\title{
Some Results on the $q$-Beta Function
}

\author{
EMRAH YILDIRIM AND İNCI EGE
}

ABstract. In this article we find some results on the $q$-analogue of the beta function via using the concepts of neutrix and neutrix limit.

\section{INTRODUCTION}

The $q$-beta function $B_{q}(x, y)$ is defined for $x, y>0$ by

$$
B_{q}(x, y)=\int_{0}^{1} t^{x-1}(1-q t)_{q}^{y-1} d_{q} t
$$

see [1], and the integral diverging for $x, y \leq 0$.

By using the concepts of neutrix and neutrix limit see [2], the $q$-beta function $B_{q}(x, y)$ is defined for all values of $x$ and $y$, see [3] and its derivative by first parameter is defined as

$$
B_{q}^{(r, 0)}(x, y)=\underset{\varepsilon \rightarrow 0}{\mathrm{~N}-\lim _{\varepsilon}} \int_{\varepsilon}^{1-\varepsilon} t^{x-1} \ln ^{r} t(1-q t)_{q}^{y-1}
$$

for $x, y \neq 0,-1,-2, \ldots$ and $r \in \mathbb{N}$, see [4].

In the following, we let $\mathcal{N}$ be the neutrix having domain $N^{\prime}=\{\varepsilon: 0<$ $\varepsilon<\infty\}$ and range $N^{\prime \prime}$ the real numbers, with the negligible functions being finite linear sums of the functions

$$
\varepsilon^{\lambda} \ln ^{r-1}, \ln ^{r} \varepsilon, \quad(\lambda<0, r \in \mathbb{N})
$$

and all functions of $\varepsilon$ which converge to zero in the normal sense as $\varepsilon$ tends to zero.

One of the differences between quantum and ordinary calculus is that the derivative of the product of two functions is not symmetric. Because of this, the $q$-integration by parts can be given in two different ways. First one was given in [1] and the second one which we will use throughout in the paper, is that

$$
\int_{a}^{b} f(q x) d_{q} g(x)=f(b) g(b)-f(a) g(a)-\int_{a}^{b} g(x) d_{q} f(x) .
$$

2010 Mathematics Subject Classification. Primary: 33D05; Secondary: 05A30, 33B15.

Key words and phrases. neutrix; neutrix limit; beta Function, $q$-beta function, $q$ integral, $q$-integration, $q$-derivative. 
In this work we find some results for the derivative of the $q$-beta function.

\section{Main Results}

Before we obtain main results, we need the following lemma which can be shown easily by induction.

Lemma 2.1. We have for $r=0,1,2, \ldots$

$$
\mathrm{N}-\lim _{\varepsilon \rightarrow 0} \int_{\varepsilon}^{1-\varepsilon} t^{-1} \ln ^{r} t d_{q} t=0
$$

and the neutrix limit of the integral

$$
\int_{\varepsilon}^{1-\varepsilon} t^{j} \ln ^{r} t d_{q} t
$$

exists as $\varepsilon \rightarrow 0$ for $j \neq-1$ and $r=0,1,2, \ldots$

Theorem 2.1. For $r, n=1,2, \ldots$ we have

$$
\begin{gathered}
B_{q}^{(r, 0)}(0,1)=0 \\
B_{q}^{(r, 0)}(0,0)=B_{q}^{(r, 0)}(1,0) \\
B_{q}^{(r, 0)}(0, n+1)=-\left(q^{n}-1\right) \sum_{j=0}^{r}\left(\begin{array}{l}
r \\
j
\end{array}\right) \ln ^{r-j-1} q^{-1} B_{q}^{j+1,0}(1, n) \\
-\sum_{j=0}^{r-1}\left(\begin{array}{l}
r \\
j
\end{array}\right) \ln ^{r-j-1} q^{-1} B_{q}^{j+1,0}(0, n+1) \\
B_{q}^{(r, 0)}(-1,0)=B_{q}^{(r, 0)}(-1,1)+B_{q}^{(r, 0)}(1,0)
\end{gathered}
$$

$B_{q}^{(r, 0)}(-n-1,1)=\frac{\ln ^{r} q^{-1}}{[-n-1]}+\frac{1}{q^{-n-1}-1} \sum_{j=0}^{r-1}\left(\begin{array}{l}r \\ j\end{array}\right) \ln ^{r-j} q^{-1} B_{q}^{(j, 0)}(-n-1,1)$

Proof. It follows from the definition of $q$-derivative that

$$
\int_{\varepsilon}^{1-\varepsilon} t^{-1} \ln t d_{q} t=\frac{1}{2} \frac{q-1}{\ln q} \ln (1-\varepsilon) \ln q^{-1}(1-\varepsilon)-\ln \varepsilon \ln q^{-1} \varepsilon,
$$

and

$$
\mathrm{N}-\lim _{\varepsilon \rightarrow 0} \int_{\varepsilon}^{1-\varepsilon} t^{-1} \ln t d_{q} t=0
$$

so equation (3) follows.

Now for (4), we have

$$
B_{q}^{(r, 0)}(0,0)=\mathrm{N}-\lim _{\varepsilon \rightarrow 0} \int_{\varepsilon}^{1-\varepsilon} t^{-1} \ln ^{r} t(1-q t)_{q}^{-1} d_{q} t
$$




$$
\begin{aligned}
& =\underset{\varepsilon \rightarrow 0}{\mathrm{~N}-\lim _{\varepsilon}} \int_{\varepsilon}^{1-\varepsilon} t^{-1} \ln ^{r} t \frac{1}{1-t} d_{q} t \\
& =\underset{\varepsilon \rightarrow 0}{\mathrm{~N}-\lim _{\varepsilon}} \int_{\varepsilon}^{1-\varepsilon} t^{-1} \ln ^{r} t d_{q} t+\mathrm{N}-\lim _{\varepsilon \rightarrow 0} \int_{\varepsilon}^{1-\varepsilon} \frac{\ln ^{r} t}{1-t} d_{q} t \\
& =B_{q}^{(r, 0)}(1,0)
\end{aligned}
$$

for $r=1,2,3, \ldots$.

For this time, let us take $f(q t)=\ln ^{r} t(1-q t)_{q}^{n}$ and $g(t)=\frac{q-1}{\ln q} \ln t$. Then using $q$-integration by parts, we obtain

$$
\begin{aligned}
& \int_{\varepsilon}^{1-\varepsilon} t^{-1} \ln ^{r} t(1-q t)_{q}^{n} d_{q} t \\
& =\underset{\varepsilon \rightarrow 0}{\mathrm{~N}-\lim _{\varepsilon \rightarrow 0}} \frac{q-1}{\ln q} \ln (1-\varepsilon) \ln ^{r}\left(q^{-1}(1-\varepsilon)\right)(1-(1-\varepsilon))_{q}^{n} \\
& -\frac{q-1}{\ln q} \ln (\varepsilon) \ln ^{r}\left(q^{-1} \varepsilon\right)(1-\varepsilon)_{q}^{n} \\
& -\frac{q-1}{\ln q} \int_{\varepsilon}^{1-\varepsilon} \ln t\left[-[n] \sum_{j=0}^{r}\left(\begin{array}{l}
r \\
j
\end{array}\right) \ln ^{r-j} q^{-1} \ln ^{j} t(1-q t)_{q}^{n-1}\right. \\
& \left.-\frac{(1-q t)_{q}^{n}}{q-1} \sum_{j=0}^{r-1}\left(\begin{array}{l}
r \\
j
\end{array}\right) \ln ^{r-j} q^{-1} t^{-1} \ln ^{j} t\right] d_{q} t \\
& =\underset{\varepsilon \rightarrow 0}{\mathrm{~N}-\lim _{\varepsilon \rightarrow 0}} \frac{q-1}{\ln q} \ln (1-\varepsilon) \ln ^{r}\left(q^{-1}(1-\varepsilon)\right)(1-(1-\varepsilon))_{q}^{n} \\
& -\frac{q-1}{\ln q} \ln (\varepsilon) \ln ^{r}\left(q^{-1} \varepsilon\right)(1-\varepsilon)_{q}^{n} \\
& -\left(q^{n}-1\right) \sum_{j=0}^{r}\left(\begin{array}{l}
r \\
j
\end{array}\right) \ln ^{r-j-1} q^{-1} \int_{\varepsilon}^{1-\varepsilon} \ln ^{j+1} t(1-q t)_{q}^{n-1} d_{q} t \\
& -\sum_{j=0}^{r-1}\left(\begin{array}{l}
r \\
j
\end{array}\right) \ln ^{r-j-1} q^{-1} \int_{\varepsilon}^{1-\varepsilon} t^{-1} \ln ^{j+1} t(1-q t)_{q}^{n} d_{q} t .
\end{aligned}
$$

Taking the neutrix limit of both sides gives equation (5).

Next

$$
\begin{aligned}
& B_{q}^{(r, 0)}(-1,0)=\underset{\varepsilon \rightarrow 0}{\mathrm{~N}-\lim _{\varepsilon}} \int_{\varepsilon}^{1-\varepsilon} t^{-2} \ln ^{r} t(1-q t)_{q}^{-1} d_{q} t \\
= & \underset{\varepsilon \rightarrow 0}{\mathrm{~N}-\lim _{\varepsilon}} \int_{\varepsilon}^{1-\varepsilon} t^{-2} \ln ^{r} t \frac{1}{1-t} d_{q} t \\
= & \underset{\varepsilon \rightarrow 0}{\mathrm{~N}-\lim _{\varepsilon \rightarrow 0}}\left\{\int_{\varepsilon}^{1-\varepsilon} t^{-2} \ln ^{r} t d_{q} t+\int_{\varepsilon}^{1-\varepsilon} t^{-1} \ln ^{r} t d_{q} t+\int_{\varepsilon}^{1-\varepsilon} \ln ^{r} t \frac{1}{1-t} d_{q} t\right\},
\end{aligned}
$$


and since the neutrix limit of second integral is zero in the last equation, we obtain

$$
B_{q}^{(r, 0)}(-1,0)=B_{q}^{(r, 0)}(-1,1)+B_{q}^{(r, 0)}(1,0) .
$$

In particular, for $r=1$ we have

$$
\begin{aligned}
& B_{q}^{(1,0)}(-1,0)=\mathrm{N}-\lim _{\varepsilon \rightarrow 0} \int_{\varepsilon}^{1-\varepsilon} t^{-2} \ln t(1-q t)_{q}^{-1} d_{q} t \\
= & \mathrm{N}-\lim _{\varepsilon \rightarrow 0} \int_{\varepsilon}^{1-\varepsilon} t^{-2} \ln t \frac{1}{1-t} d_{q} t \\
= & \mathrm{N}-\lim _{\varepsilon \rightarrow 0}\left\{\int_{\varepsilon}^{1-\varepsilon} t^{-2} \ln t d_{q} t+\int_{\varepsilon}^{1-\varepsilon} t^{-1} \ln t d_{q} t+\int_{\varepsilon}^{1-\varepsilon} \ln t \frac{1}{1-t} d_{q} t\right\} \\
= & -\frac{q \ln q}{[1]^{2}(q-1)}+B_{q}^{(1,0)}(1,0) .
\end{aligned}
$$

To obtain equation (7), let us take $f(t)$ and $g(t)$ as $f(q t)=\ln ^{r} q^{-1} t$ and $g(t)=\frac{t^{-n-1}}{[-n-1]}$ respectively and then we get

$$
\begin{aligned}
& B_{q}^{(r, 0)}(-n-1,1)=\mathrm{N}-\lim _{\varepsilon \rightarrow 0} \int_{\varepsilon}^{1-\varepsilon} t^{-n-2} \ln ^{r} q^{-1} t d_{q} t \\
= & \mathrm{N}-\lim _{\varepsilon \rightarrow 0} \frac{(1-\varepsilon)^{-n-1}}{[-n-1]} \ln ^{r} q^{-1}(1-\varepsilon)-\frac{\varepsilon^{-n-1}}{[-n-1]} \ln ^{r} q^{-1} \varepsilon \\
& +\frac{1}{[-n-1](q-1)} \sum_{j=0}^{r-1}\left(\begin{array}{l}
r \\
j
\end{array}\right) \ln ^{r-j} q^{-1} \int_{\varepsilon}^{1-\varepsilon} t^{-n-2} \ln ^{j} t d_{q} t .
\end{aligned}
$$

This completes the proof.

Also it should be noted that by taking $f(t)=\ln ^{r} t$ and $g(t)=\frac{t^{-n-1}}{[-n-1]}$ and using $q$-integration by parts in [1] we have

$$
\begin{gathered}
\int_{\varepsilon}^{1-\varepsilon} t^{-n-2} \ln ^{r} q^{-1} t d_{q} t=\frac{(1-\varepsilon)^{-n-1}}{[-n-1]} \ln ^{r}(1-\varepsilon)-\frac{\varepsilon^{-n-1} \ln ^{r} \varepsilon}{[-n-1]} \\
-\frac{1}{[-n-1](q-1)} \sum_{j=0}^{r-1}\left(\begin{array}{c}
r \\
j
\end{array}\right) \ln ^{r-j} q \int_{\varepsilon}^{1-\varepsilon}(q t)^{-n-1} t^{-1} \ln ^{j} t d_{q} t
\end{gathered}
$$

and taking the neutrix limit on both sides we get

$$
B_{q}^{(r, 0)}(-n-1,1)=\frac{1}{q^{n+1}-1} \sum_{j=0}^{r-1}\left(\begin{array}{l}
r \\
j
\end{array}\right) \ln ^{r-j} q B_{q}^{(j, 0)}(-n-1,1) .
$$

If we use property of the $q$-analogue of $(1-t)^{n}$ on $B_{q}^{(r, 0)}(0, n)$ we get

$$
B_{q}^{(r, 0)}(0, n)=\underset{\varepsilon \rightarrow 0}{\mathrm{~N}-\lim _{\varepsilon}} \int_{\varepsilon}^{1} t^{-1} \ln ^{r} t(1-q t)_{q}^{n-1} d_{q} t
$$




$$
\begin{aligned}
& =\underset{\varepsilon \rightarrow 0}{\mathrm{~N}-\lim _{\varepsilon}} \int_{\varepsilon}^{1} t^{-1} \ln ^{r} t(1-q t)_{q}^{n-2}\left(1-q^{n-1} t\right) d_{q} t \\
& =\underset{\varepsilon \rightarrow 0}{\mathrm{~N}-\lim _{\varepsilon}} \int_{\varepsilon}^{1} t^{-1} \ln ^{r} t(1-q t)_{q}^{n-2} d_{q} t-q^{n-1} \int_{\varepsilon}^{1} \ln ^{r} t d_{q} t
\end{aligned}
$$

and so

$$
B_{q}^{(r, 0)}(0, n)=\underset{\varepsilon \rightarrow 0}{\mathrm{~N}-\lim _{\varepsilon}} \int_{\varepsilon}^{1} t^{-1} \ln ^{r} t d_{q} t-\sum_{j=1}^{n-1} q^{j} \int_{\varepsilon}^{1} \ln ^{r} d_{q} t
$$

Hence by lemma 2.1 we get

$$
B_{q}^{(r, 0)}(0, n)=-\sum_{j=1}^{n-1} q^{j} B_{q}^{(r, 0)}(1,1)
$$

We can similarly obtain that

$$
B_{q}^{(r, 0)}(1, n)=B_{q}^{(r, 0)}(1,1)-\sum_{j=1}^{n-1} q^{j} B_{q}^{(r, 0)}(2,1)
$$

Now we generalize equation (6) as follows.

\section{Theorem 2.2.}

$$
B_{q}^{(r, 0)}(-n, 0)=B_{q}^{(r, 0)}(1,0)+\sum_{j=2}^{n+1} B_{q}^{(r, 0)}(-j+1,1)
$$

for $r, n=1,2, \ldots$

Proof. If we apply $q$-integration by parts on the definition of beta function, then we get

$$
\begin{aligned}
B_{q}^{(r, 0)}(-n, 0) & =\underset{\varepsilon \rightarrow 0}{\mathrm{~N}-\lim _{\varepsilon}} \int_{\varepsilon}^{1-\varepsilon} t^{-n-1} \ln ^{r} t(1-q t)_{q}^{-1} d_{q} t \\
= & \mathrm{N}-\lim _{\varepsilon \rightarrow 0}\left\{\int_{\varepsilon}^{1-\varepsilon} \ln ^{r} t(1-q t)_{q}^{-1} d_{q} t+\int_{\varepsilon}^{1-\varepsilon} t^{-1} \ln ^{r} t d_{q} t\right. \\
& \left.+\sum_{j=2}^{n+1} \int_{\varepsilon}^{1-\varepsilon} t^{-j} \ln ^{r} t d_{q} t\right\} .
\end{aligned}
$$

Hence by lemma 2.1, the proof is completed.

In next theorem, the constants $c_{r, n}(m)$ of the expansion of the terms

$$
(1-\varepsilon)^{n-1} \ln ^{r}(1-\varepsilon)=\sum_{i=0}^{\infty} c_{r, n}(i) \varepsilon^{i}
$$


can be presented as

$$
c_{r, n}(m)= \begin{cases}0, & m<r \\ (-1)^{m}, & m=r \\ n-m, & m=r+1\end{cases}
$$

for $r, m=1,2, \ldots$

Theorem 2.3. We have

$$
\begin{aligned}
B_{q}^{(r, 0)}(n,-m)= & \frac{c_{r, n}(m)}{m}+\frac{[n-1]}{[-m]} B_{q}^{(n, 0)}(n-1,-m+1) \\
& +\frac{q^{n-1}}{[-m]} \sum_{k=0}^{r-1}\left(\begin{array}{l}
r \\
k
\end{array}\right) \ln ^{r-k} q B_{q}^{(k, 0)}(n-1,-m+1)
\end{aligned}
$$

for $r, m=1,2, \ldots$ and $n=2,3, \ldots$.

Proof. Since we have

$$
\begin{aligned}
& \int_{\varepsilon}^{1-\varepsilon} t^{n-1} \ln ^{r} t(1-q t)_{q}^{-m-1} d_{q} t \\
& =-\left.\frac{t^{n-1} \ln ^{r} t(1-t)_{q}^{-m}}{[-m]}\right|_{\varepsilon} ^{1-\varepsilon}-\frac{1}{[-m]}\left\{[n-1] \int_{\varepsilon}^{1-\varepsilon} t^{n-2} \ln ^{r} t(1-q t)_{q}^{-m} d_{q} t\right. \\
& \left.+\sum_{k=0}^{r-1}\left(\begin{array}{l}
r \\
k
\end{array}\right) \ln ^{r-k} t \int_{\varepsilon}^{1-\varepsilon}(q t)^{n-1} \ln ^{k} t(1-q t)_{q}^{-m} d_{q} t\right\} \\
& =-\frac{(1-\varepsilon)^{n-1} \ln ^{r}(1-\varepsilon) \varepsilon^{-m}}{[-m]}+\frac{\varepsilon^{n-1} \ln ^{r} \varepsilon(1-\varepsilon)_{q}^{-m}}{[n-1]} \\
& +\frac{[-m]}{[n} \int_{\varepsilon}^{1-\varepsilon} t^{n-2} \ln ^{r} t(1-q t)_{q}^{-m} d_{q} t \\
& +\frac{q^{n-1}}{[-m]} \sum_{k=0}^{r-1}\left(\begin{array}{l}
r \\
k
\end{array}\right) \ln ^{r-k} t \int_{\varepsilon}^{1-\varepsilon} t^{n-1} \ln ^{k} t(1-q t)_{q}^{-m} d_{q} t
\end{aligned}
$$

the proof is completed by taking the neutrix limit of both side of the last equation.

Finally, we note that all results obtained in the paper are in agreement with the results given in [5-10] as $q$ tends 1 .

\section{REFERENCES}

[1] V. Kac, and P. Cheung, Quantum calculus, Springer, 2002.

[2] J.G. van der Corput, Introduction to the neutrix calculus, J. Analyse Math., Vol.7(60) (1959),291-398.

[3] İ Ege, On defining the q-beta function for negative integers, Filomat, Vol.27(2) (2013), 251-260. 
[4] A. Salem, Generalized q-integrals via neutrices: Application to the q-beta function, Filomat, Vol. 27(8) (2013), 1473-1483.

[5] E. Özçă̆, B. Fisher, On partial derivatives of the Beta function, Rostock Math. Kolloq.,Vol.45 (1991), 43-56.

[6] B. Fisher, Y. Kuribyashi, Neutrices and the beta function, Rostock Math Kolloq., Vol.32 (1987), 1-22.

[7] B. Fisher, Results on the beta function, , Sarajevo Journal Of Mathematics, Vol.9(21) (2013), 101-108.

[8] F. Al-Sirehy, and B. Fisher, Further results on the beta function and the incomplete beta function, Applied Mathematical Sciences, Vol.7(70) (2013), 3489-3495.

[9] F. Al-Sirehy,and B. Fisher, Results on the beta function and the incomplete beta function, International Journal of Applied Mathematics, Vol.26(2) (2013), 191-201.

[10] F. Al-Sirehy,and B. Fisher, Evaluation of the beta function, International Journal of Applied Mathematics, Vol.26(1), (2013), 59-70.

\section{EMRAH YILDIRIM}

Department of Mathematics

Faculty of Art and Science

Adnan Menderes University

AYDIN

TURKEY

E-mail address: emrahyildirim@adu.edu.tr

\section{İNCI EGE}

Department of Mathematics

Faculty of Art and Science

Adnan Menderes University

AYDIN

TURKEY

E-mail address: iege@adu.edu.tr 